\title{
Proteomics analysis of the hypothalamus in spontaneously hypertensive rats treated with twirling reinforcing manipulation, twirling reducing manipulation or electroacupuncture
}

\author{
JINGRONG LIANG $^{1}$, JIAOJUAN WU ${ }^{1}$, XUDONG ZHANG $^{1}$, XIAOMIN HAO $^{1}$, TIANXIAO ZENG ${ }^{1}$,

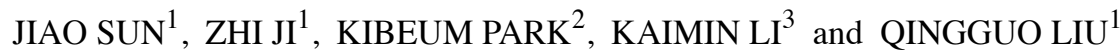 \\ ${ }^{1}$ School of Acupuncture-Moxibustion and Tuina, Beijing University of Chinese Medicine, Beijing 100029; \\ ${ }^{2}$ Shenzhen New Element Clinic, Shenzhen Bay Ecological Science and Technology Park, Shenzhen, Guangdong 518000; \\ ${ }^{3}$ School of Biological Science and Medical Engineering, Beihang University, Beijing 100191, P.R. China
}

Received February 3, 2020; Accepted June 17, 2020

DOI: $10.3892 /$ etm.2021.9812

\begin{abstract}
Hypertension is one of the primary risk factors for cardiovascular diseases. Numerous proteins serve a critical role in hypertension. Acupuncture has been widely used as a treatment for hypertension in China. The results of the current study suggested that electroacupuncture (EA), twirling reinforcing manipulation (TRFM) and twirling reducing manipulation (TRDM) may be useful in the treatment of hypertension. Additionally, proteome analysis of spontaneously hypertensive rats treated with EA, TRFM and TRDM was performed. There were 117 (EA group), 61 (TRFM group) and 86 (TRDM group) differentially expressed proteins (DEPs) identified in the respective experimental groups compared with the model group. Moreover, parallel reaction monitoring assays were used to validate the reliability of the DEPs. The majority of the results were consistent with previous proteomics results, in particular that for expression of neudesin neurotrophic factor (NENF). NENF may potentially represent an antihypertensive drug target.
\end{abstract}

\section{Introduction}

Hypertension is a major public health issue, affecting $>1.3$ billion people worldwide (1) and $>90 \%$ of patients with hypertension exhibit essential hypertension (EH) (2). EH is a multifactorial chronic disease that is influenced by genetic factors, obesity, stress, diet and other factors (3-6). The primary regions affected by hypertension are the heart, brain, kidneys and blood vessels (7). Effective and stable control

Correspondence to: Professor Qingguo Liu, School of Acupuncture-Moxibustion and Tuina, Beijing University of Chinese Medicine, 11 Beisanhuan Dong Lu, Beijing 100029, P.R. China E-mail: liuqingguo888@vip.sina.com

Key words: proteomics, spontaneously hypertensive rats, acupuncture, hypothalamus, parallel reaction monitoring of blood pressure is urgently required for public health. Hypertension is primarily treated with drugs (8), which have certain disadvantages, including poor patient compliance (9), adverse reactions (10) and unstable curative effects (11).

Numerous studies have demonstrated that acupuncture lowers blood pressure (12-14). Acupuncture has been practiced in China for $>3,000$ years and spread to Europe and North America between the 16 and 19th centuries (15). Huang Di Nei Jing (Huangdi's Inner Classic), an ancient Chinese medical book, recorded the use of acupuncture treatment for hypertension 2,000 years ago (16). Acupuncture treatment has been reported to have a therapeutic effect on various diseases, particularly hypertension $(17,18)$. Previous studies have demonstrated that reinforcing and reducing manipulation was able to decrease blood pressure via multiple biological processes, including upregulation of nitric oxide synthase and cyclic guanosine monophosphate in arterial tissues (19), the brain network (20) and cell signal transduction pathways (21). Positron emission tomography functional imaging the brains of rats who had receiving twirling reinforcing and reducing manipulation indicated that these acupuncture manipulations had central effects on mechanisms such as cerebral glucose metabolism (22). Different acupuncture manipulations activate different brain areas and these areas produce neurotransmitters that regulate blood pressure (22). These results indicated that the brain exhibited core response to acupuncture manipulations and that neural protein expression, including the expression of related neurotransmitters, was impacted. These neurotransmitters acted on target organs to reduce blood pressure. A previous proteomics study demonstrated that the expression of various proteins other than neurotransmitters was altered in rat medullas following acupuncture treatment (23). Furthermore, a previous study reported that acupuncture affects multiple systems and targets, including the renin-angiotensin-aldosterone system, the neuroendocrine system, the vascular endothelium and oxidative stress (24). The cross-effect of these targets may be the mechanism involved in lowering blood pressure (25).

Twirling reinforcing manipulation, twirling reducing manipulation and electroacupuncture are different acupuncture 
techniques and may produce effects on the brain (26). The hypothalamus serves an important role in the development and maintenance of hypertension (27). Taichong (LR3) is located between the first and the second metatarsal bone of dorsal foot (28) and is the most commonly used acupuncture point in acupuncture for hypertension (29). The current study hypothesized that electroacupuncture, twirling reinforcing manipulation and twirling reducing manipulation may activate the hypothalamus to release neurotransmitters by influencing protein expression profile, thus regulating blood pressure and that different acupuncture manipulations have different protein expression patterns, leading to differences in the effects on blood pressure. Spontaneously hypertensive rats (SHRs) were established as an essential hypertension animal model by inbreeding of Wistar-Kyoto (WKY) rats, as previously described (30). The aim of the current study was to determine the potential antihypertensive effect of different acupuncture manipulations in lowering blood pressure.

\section{Materials and methods}

Animals. All animal experimental procedures were conducted in accordance with the World Health Organization's International Guiding Principles for Biomedical Research Involving Animals (31) and were approved by the Animal Care and Use Committee of Beijing University of Chinese Medicine, Beijing, China (permit no. BUCM-3-2016090301-3003). All rats were housed with 4 individuals per clean cage, with free access to water and food on a $12 \mathrm{~h}$ light/dark cycle at a humidity of $50-60 \%$ and at a temperature of $18-22^{\circ} \mathrm{C}$ during the process. A total of 32 SHR and 8 WKY male rats (age, 9 weeks; weight, $215 \pm 20 \mathrm{~g}$ ) were obtained from Beijing Vital River Laboratory Animal Technology Co., Ltd. SHRs were randomly divided into the model $(\mathrm{M})$, twirling reinforcing manipulation (TRFM), twirling reducing manipulation (TRDM) and electroacupuncture (EA) groups ( $n=8 /$ group). In order to establish the model male WKY rats with continuous systolic blood pressures of $150-175 \mathrm{mmHg}$ were mated with female WKY rats with systolic blood pressures of $130-140 \mathrm{mmHg}$ by Beijing Vital River Laboratory Animal Technology Co., Ltd. Offspring with systolic blood pressures $>150 \mathrm{mmHg}$ were obtained. Rats with high blood pressure were selected for inbreeding and the stable inheritance of hypertension was obtained by selective inbreeding for 20 generations and the SHR model was established $(30,32)$. WKY rats were used as the normotensive control group of SHR and the control strain of hypertensive rats, as previously described (33).

Sample preparation. All acupuncture manipulations were performed following 1 week of adaptation. The blood pressure of the WKY rats was relatively stable. SHRs were easily irritated and hyperactive; however, they were relatively quiet in the morning. The systolic blood pressure in the caudal artery was measured by two experienced technicians at a controlled temperature of $\left(20 \pm 2^{\circ} \mathrm{C}\right)$. Each rat was gently placed in a restrainer and its tail was fixed using the rat-tail fixing facility. The ventral portion of each rat was placed on a heat pad, while the blood pressure measurement cuffs were put in place. Once a batch of 2 rats was in place, calm rats were preheated at $36^{\circ} \mathrm{C}$ for $10 \mathrm{~min}$, after which their systolic blood pressure was measured with a non-invasive blood pressure instrument (BP-6; Chengdu TME Technology Co., Ltd.) and recorded by the pulse recording sensor while the rats were quiet and conscious. Each rat was measured three times and the mean value of the three times was taken as the systolic pressure. The systolic blood pressure of the rats was measured between 8:00 and 12:00 a.m. every two days between day 1 (one day prior the acupuncture experiment) and day 15. Acupuncture at Taichong (LR3) was performed between 2:00 and 4:00 p.m. every day for 2 weeks. The 8 WKY (group WKY) and 8 SHRs (group M) did not undergo acupuncture; however, they were handled and held for $20 \mathrm{~min}$ in the same restrainer. Acupuncture on the SHRs in group TRFM , TRDM and EA was performed according to previous published studies $(22,33)$. Briefly, acupuncture needles were inserted to LR3 loci with respective acupuncture manipulations. For TRFM, the manipulation was performed with the forward thumb exerting heavy force, while the backward thumb exerted light force; meanwhile, for TRDM, the manipulation was conducted with the forward thumb exerting light fore with the backward thumb exerting with heavy force. The frequency and angle used were the same as a previous study (34). EA was conducted according to another previous study (35). The rats were treated once per day for 14 days. The same acupuncturist performed all acupuncture and sham treatments.

Hypothalami were collected for proteomics analysis. All experimental rats were sacrificed following anesthesia with an intraperitoneal injection of $3 \%$ pentobarbital sodium $(30 \mathrm{mg} / \mathrm{kg})$. The anesthetic regimen resulted in adequate anesthesia (Pinna reflex and pedal reflex were tested.) in all rats within $10 \mathrm{~min}(36,37)$. Rats were sacrificed by cervical dislocation (38). The hypothalamus was isolated from each rat brain using tweezers and transferred to liquid nitrogen for preservation.

Protein extraction and digestion. Hypothalami were homogenized three times at speed 6 for $40 \mathrm{sec}$ in $8 \mathrm{M}$ urea supplemented with cOmplete ${ }^{\mathrm{TM}}$ protease inhibitor cocktail (Roche Diagnostics) using a FastPrep instrument (MP Biochemicals, Inc.; Thermo Fisher Scientific, Inc.). The supernatant containing total protein was collected following centrifugation at $13,000 \mathrm{x} \mathrm{g}$ for $30 \mathrm{~min}$ at $4^{\circ} \mathrm{C}$. Concentrations were determined using a BCA assay kit (Thermo Fisher Scientific, Inc.) according to the manufacturer's protocol. A total of $150 \mu \mathrm{g}$ of protein from each sample was reduced with $10 \mathrm{mM}$ Tris-(2-carboxyethyl) phosphine (Thermo Fisher Scientific, Inc.) at $37^{\circ} \mathrm{C}$ for $60 \mathrm{~min}$ and alkylated with $40 \mathrm{mM}$ iodoacetamide (cat. no. A3221; Sigma-Aldrich; Merck, KGaA) in the dark at room temperature for $30 \mathrm{~min}$. Protein samples were collected using acetone precipitation. A volume of $400 \mu \mathrm{l}$ acetone was added to each sample which was then stored at $-20^{\circ} \mathrm{C}$ for $2 \mathrm{~h}$. Proteins was collected by centrifugation at $16,000 \mathrm{x} \mathrm{g}$ at $4^{\circ} \mathrm{C}$ for $20 \mathrm{~min}$. Then the protein samples were digested with trypsin (Promega Corporation) at $37^{\circ} \mathrm{C}$ overnight. The peptides were desalted on Sep-Pak tC18 cartridges (Waters Corporation), according to the manufacturer's protocol.

Label-free proteomics analysis. Tryptic peptides were analyzed on a Q-Exactive mass spectrometer (Thermo Fisher Scientific, Inc.) coupled to an Easy-nLC 1200 nanoflow 
liquid chromatography system (Thermo Fisher Scientific, Inc.). The dried peptides were redissolved with Solvent A [2\% acetonitrile (ACN) and $0.1 \%$ trifluoroacetic acid (TFA; cat. no. 28903; Thermo Fisher Scientific, Inc.) in water] and $2 \mu \mathrm{g}$ was loaded onto a C18 column $(1.9 \mu \mathrm{m} ; 75 \times 25 \mathrm{~cm}$; Thermo Fisher Scientific, Inc.) and separated with a gradient of 5-38\% Solvent B (80\% ACN and $0.1 \%$ formic acid at a flow rate of $300 \mathrm{nl} / \mathrm{min}$. The data-dependent acquisition mode was used for the mass spectrometry (MS) for $120 \mathrm{~min}$ at room temperature. Full mass scans $(350-1800 \mathrm{~m} / \mathrm{z})$ were acquired at a resolution of 70,000 and the top 20 most abundant precursor ions were selected for higher-energy collisional dissociation fragmentation at a resolution of 17,500. The dynamic exclusion was $18 \mathrm{sec}$.

Proteome Discoverer software (version 2.2; Thermo Fisher Scientific, Inc.) was used for protein identification against the Rattus norvegicus UniProt database (https://www. uniprot.org/uniprot/?query=reviewed:yes\%20taxonomy:10114; 28-08-2018). Cysteine reduced with iodoacetamide was set as the static modification and the oxidation of methionine and acetylation of the protein N-terminus were set as the dynamic modifications. The maximum missed cleavage sites and the precursor mass tolerance were set to 2 and $10 \mathrm{ppm}$, respectively. The cutoff of the global false discovery rate was set to 0.01 at the peptide level.

Parallel reaction monitoring (PRM)-MS validation. Protein abundance differences obtained in the label-free proteomics analysis were confirmed using PRM assays. Independent retention time peptides (Biognosys $A G$ ) were added to the samples, according to the manufacturer's protocol. The scheduled PRM assays were conducted on the Q-Exactive HF coupled Easy-nLC 1,200 nanoflow liquid chromatography system (Thermo Fisher Scientific, Inc.). SpectroDive 9 software (version SW-3002; Biognosys AC) was used to develop the inclusion list (Table SI) and perform data analysis using the default parameters.

Statistical analysis. Mixed ANOVA and the Bonferroni's post-hoc test was performed to detect differences in blood pressure. Label-free and PRM data were analyzed using one-way ANOVA followed Tukey's post-hoc test. The Venn diagram was drawn using $\mathrm{R}$ version 3.6.3 (VennDiagram package). Gene ontology (GO) annotation was performed using Blast2GO ${ }^{\circledR}$ (www.blast2go.com) and GOATOOLS (v0.6.10; https://github.com/tanghaibao/goatools/releases/tag/v0.6.10) was used to run the GO enrichment analysis. P-values were assessed using Fisher's exact test and adjusted following the Benjamini-Hochberg method. Qiagen Ingenuity Pathway Analysis (IPA) software (version 2019; QIAGEN, Inc.) was used for the function and network analyses of the differentially expressed proteins (DEPs, $\mathrm{P}<0.05$; fold change $\geq 1.3$ ). A z-score algorithm was used to predict the function of the DEPs. A size of 35 focus molecules, both direct and indirect relationships with Fisher's exact test were applied to run the network analysis (score=-logP-value). One-way ANOVA and Bonferroni's post-hoc test was performed for PRM data analysis. Graphs of PRM data were prepared using GraphPad Prism (version 6; GraphPad Software, Inc.). All statistical analysis was performed using SPSS (version 22; IBM Corp.)

\section{Results}

Multiple acupuncture manipulations lower blood pressure in SHRs. To study the antihypertensive effects of different acupuncture manipulations, TRFM, TRDM and EA was performed on SHRs. The systolic blood pressure of all experimental rats was measured regularly (Table I). The results demonstrated that there was no significant difference in the blood pressure measurements between the acupuncturetreated groups (TRDM, TRFM and EA) and group M (all, $\mathrm{P}>0.05$ ) on day 0 (one day prior to acupuncture manipulation). However, the blood pressure measurements of the M, TRDM, TRFM and EA groups were significantly higher compared with group WKY at all time points (all $\mathrm{P}<0.05$ ). The blood pressure measurements of the TRDM, TRFM and EA groups decreased significantly compared with the M group on days 8 , 13 and 15 (all, $\mathrm{P}<0.05$ ). All three acupuncture manipulation groups exhibited attenuated blood pressures following 15 days of treatment; however, the blood pressure measurements of the TRDM, TRFM and EA groups were reduced by varying degrees. The blood pressure measurements of the TRDM group were lower compared with the TRFM and EA groups on day 15; however, the differences were not significant (both, $\mathrm{P}>0.05)$. These results indicated that the acupuncture manipulations had a positive effect in lowering the blood pressure measurements of the SHR groups, with TRDM possibly being the most effective treatment. The blood pressure measurements of the TRDM group declined over time and there was a significant difference at day 15 vs. day $3(\mathrm{P}<0.05)$. There was a slight decrease in the blood pressure measurements in the TRDM and EA groups; however, P $>0.05$ in all cases (all the others days vs. day 0). These data indicated that TRDM, TRFM and EA lowered the blood pressures of the SHRs by varying degrees. Furthermore, the data revealed an instant and long-term protective effect of TRDM and an instant effect of TRFM and EA in SHRs.

Analyses of the proteomics data. To investigate the effect of acupuncture treatment on the central nervous system, proteomics analysis on the hypothalami of the SHRs was performed. Hypothalami from all rats were subjected to proteomics analysis through a label-free technique to obtain profiling data. The results identified 4,483 proteins (Fig. 1A, Table SII). Following this, four comparisons (M/WKY, TRFM/M, EA/M and TRDM/M) were performed to identify altered proteins. A total of 241 (M/WKY), 61 (TRFM/M), $117(\mathrm{EA} / \mathrm{M})$ and $86(\mathrm{TRDM} / \mathrm{M})$ proteins were identified as DEPs $(\mathrm{P}<0.05$; fold change $\geq 1.3$ ). Further comparisons were performed to investigate the differences and overlaps in the DEPs induced by the TRDM, TRFM and EA manipulations via Venn analysis (Fig. 1B), indicating the presence of numerous rescue effect-related proteins. GO enrichment analysis of DEPs in the M group compared with the WKY group was performed (Fig. 1C). The dysregulated proteins in groups TRFM, EA and TRDM were compared with the $\mathrm{M}$ group. Following this, GO enrichment analyses of these proteins was conducted. Extracellular exosome, Golgi apparatus and endoplasmic reticulum membrane were among the top 5 most highly enriched $(\mathrm{P}<0.05)$ terms in all of the GO enrichment analyses (Fig. 1C and D), indicating that hypertension and acupuncture 
Table I. Systolic blood pressure measurements in the rats.

\begin{tabular}{lccccc}
\hline Group & Day 0 & Day 3 & Day 8 & Day 13 & Day 15 \\
\hline WKY & $110.12 \pm 1.46$ & $111.62 \pm 2.07$ & $112.75 \pm 2.38$ & $112.25 \pm 3.24$ & $112.50 \pm 3.66$ \\
M & $165.13 \pm 1.46^{\mathrm{a}}$ & $165.25 \pm 2.25^{\mathrm{a}}$ & $170.38 \pm 2.00^{\mathrm{a}, \mathrm{c}, \mathrm{d}}$ & $173.38 \pm 3.34^{\mathrm{a}, \mathrm{c}, \mathrm{d}}$ & $174.00 \pm 2.73^{\mathrm{a}, \mathrm{c}, \mathrm{d}}$ \\
TRFM & $164.63 \pm 2.20^{\mathrm{a}}$ & $164.50 \pm 1.93^{\mathrm{a}}$ & $163.63 \pm 3.38^{\mathrm{a}, \mathrm{b}}$ & $162.62 \pm 3.74^{\mathrm{a}, \mathrm{b}}$ & $162.50 \pm 3.36^{\mathrm{a}, \mathrm{b}}$ \\
TRDM & $164.88 \pm 2.70^{\mathrm{a}}$ & $163.75 \pm 2.60^{\mathrm{a}}$ & $161.38 \pm 3.54^{\mathrm{a}, \mathrm{b}}$ & $159.88 \pm 2.53^{\mathrm{a}-\mathrm{c}}$ & $158.13 \pm 3.04^{\mathrm{a}-\mathrm{d}}$ \\
EA & $165.00 \pm 2.00^{\mathrm{a}}$ & $163.88 \pm 3.76^{\mathrm{a}}$ & $163.38 \pm 3.02^{\mathrm{a}, \mathrm{b}}$ & $162.75 \pm 3.77^{\mathrm{a}, \mathrm{b}}$ & $162.25 \pm 4.83^{\mathrm{a}, \mathrm{b}}$ \\
\hline
\end{tabular}

${ }^{\text {aP }}<0.05$ vs. the WKY group. ${ }^{\mathrm{b}} \mathrm{P}<0.05$ vs. the $\mathrm{M}$ group. ${ }^{\mathrm{c}} \mathrm{P}<0.05$ vs. Day $0 .{ }^{\mathrm{d}} \mathrm{P}<0.05$ vs. Day 3 . Day 0 denotes 1 day prior to acupuncture manipulation. Days 3, 8, 13 and 15 denote the 3rd, 8, 13 and 15th days of acupuncture manipulation. WKY, Wistar-Kyoto; M, model; TRFM, twirling reinforcing manipulation; TRDM, twirling reducing manipulation; EA, electroacupuncture.

A



C

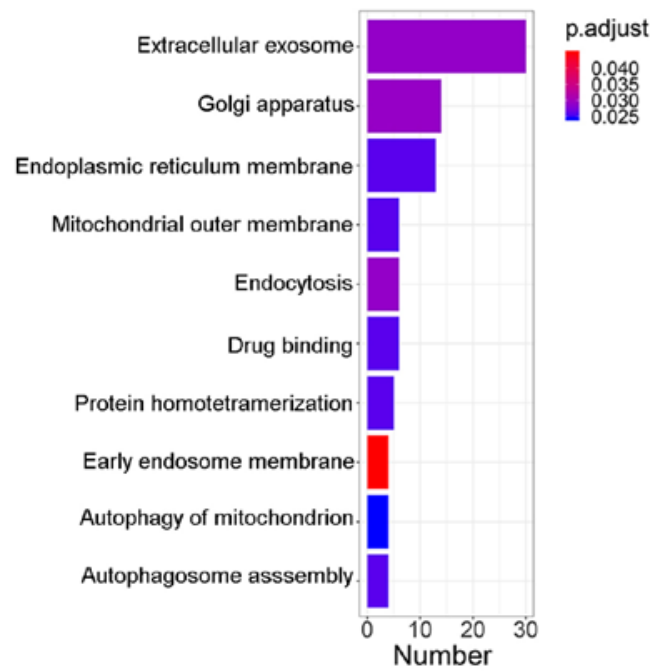

B

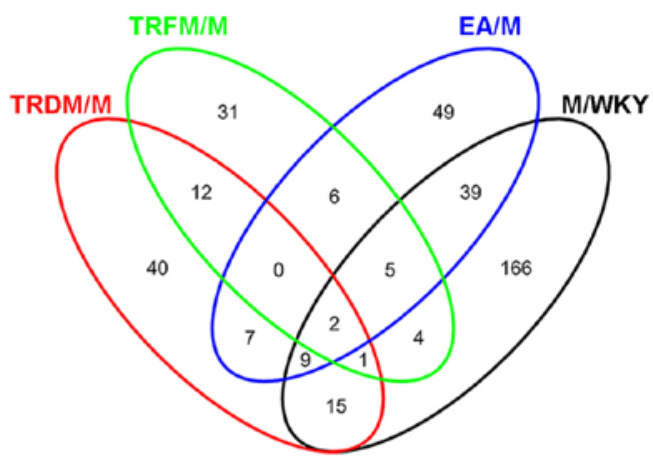

D

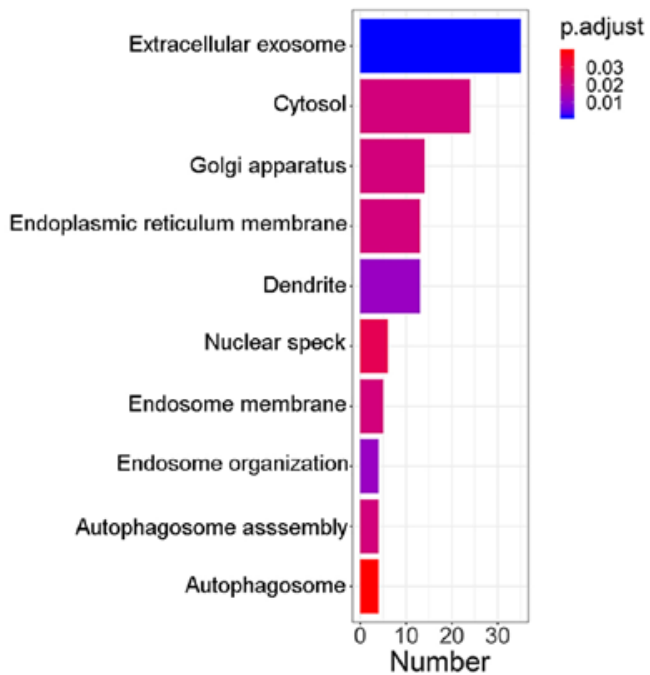

Figure 1. Bioinformatics analyses of label-free proteomics data. (A) Heatmap of differentially expressed proteins in the four comparison groups (M/WKY, TRFM/M, EA/M, TRDM/M). (B) Venn diagram of the comparison groups. (C) Top $10 \mathrm{GO}$ terms in the GO enrichment analysis of DEPs in the M groups compared with the WKY group. (D) Top $10 \mathrm{GO}$ terms in the GO enrichment analysis of all DEPs in the acupuncture groups (TRDM, TRFM and EA) compared with the M group. M, model; WKY, Wistar-Kyoto; TRFM, twirling reinforcing manipulation; EA, electroacupuncture; TRDM, twirling reducing manipulation; GO, gene ontology; DEPs, differentially expressed proteins.

manipulations affected the expression of secreted proteins in the hypothalami of the SHRs. Furthermore, other cell component terms, including symmetric synapses, and biological process terms, including endosome organization, were also significantly enriched (Table SIII). In total 11 neuron development and neuritogenesis-related proteins were identified: 
A

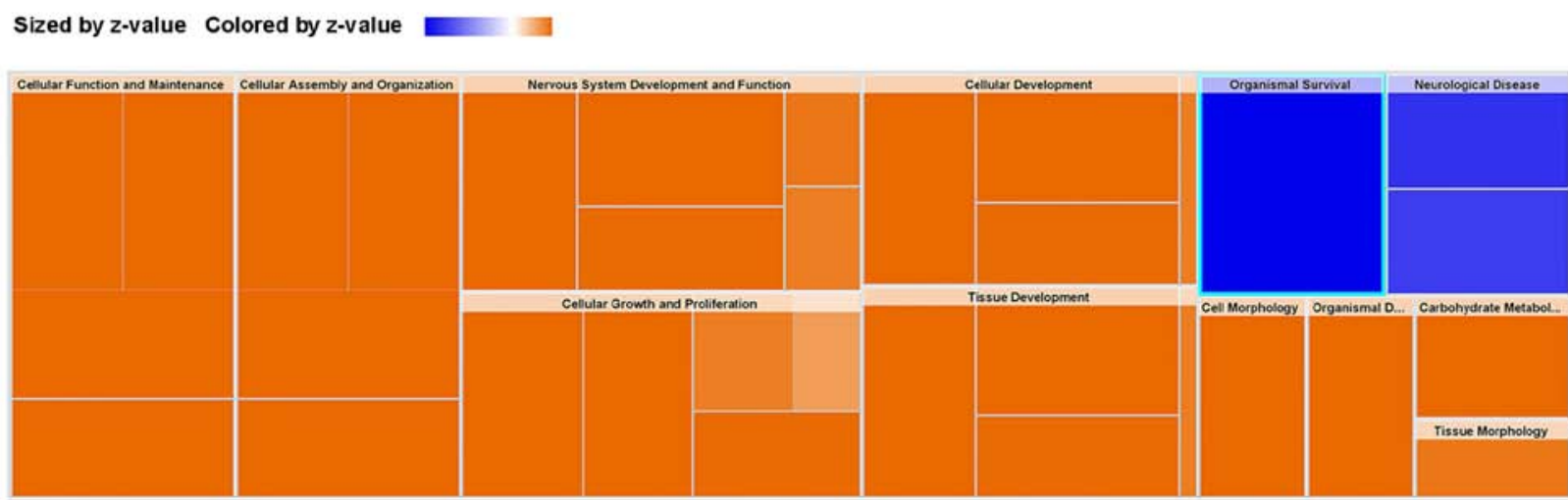

B

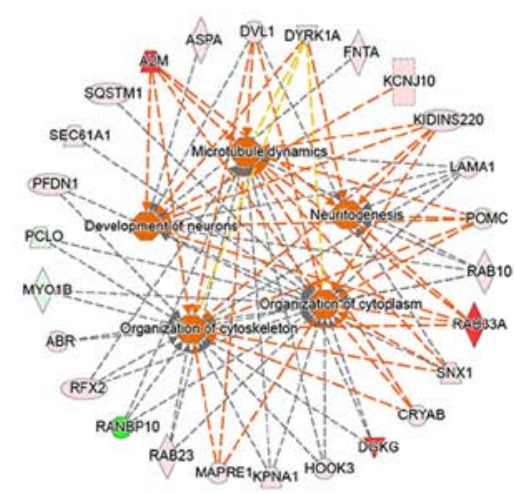

C

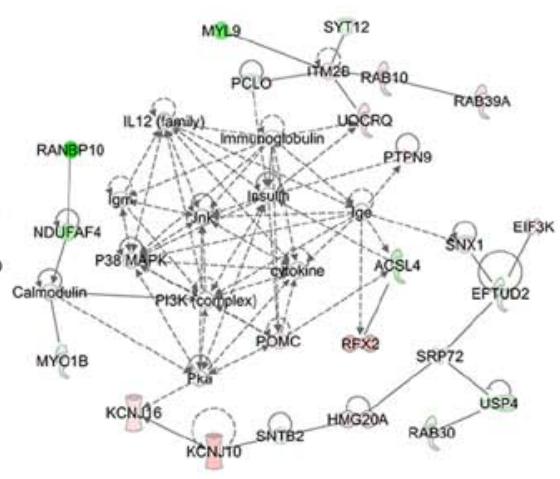

D

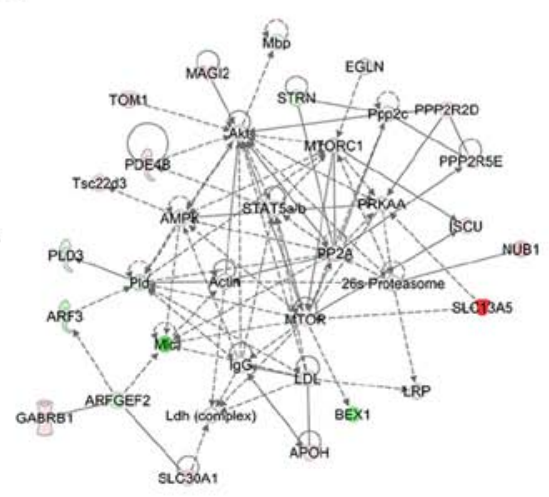

Figure 2. IPA analyses of the differentially expressed proteins. (A) Heatmap of the function and disease analysis of all differentially expressed genes (TRFM/M, $\mathrm{EA} / \mathrm{M}, \mathrm{TRDM} / \mathrm{M})$. Colors were defined by the z-scores, with orange representing upregulation and blue downregulation. Size was defined by the-log(P-value). (B) The increased effects on the cytoplasm organization, cytoskeleton organization, microtubule dynamics, neuron development and neuritogenesis, the red symbols indicate upregulated genes and green symbols downregulated genes. (C) An IPA network involved in cell morphology (Cell Morphology, Digestive System Development and Function, RNA Post-Transcriptional Modification; (D) An IPA network involved in nervous system (Nervous System Development and Function, Neurological Disease, Tissue Morphology). TRFM, twirling reinforcing manipulation; M, mode; EA, electroacupuncture; TRDM, twirling reducing manipulation. Further details of specific genes may be found in Table SIV.

A2MG, NENF, ACY2, DVL1, DYR1A, FNTA, KCJ10, KDIS, COLI, RAB10 and SNX1 (Table SIII). In summary, these results may elucidate the effect of acupuncture manipulation on the central nervous system in the SHRs.

IPA function and network analysis of the DEPs associated with acupuncture manipulation. The function and network of all the dysregulated proteins in the acupuncture-treated SHRs were evaluated using IPA software. The acupuncture manipulation-affected genes were significantly associated with multiple physiological processes (Fig. 2A), including 'increased neuronal development activity' and 'decreased neurological disease tremor and movement disorder activity' (Fig. 2B, Table SIV). The top 5 activated functions ('cytoplasm organization', 'cytoskeleton organization', 'microtubule dynamics', 'neuron development' and 'neuritogenesis') are presented. Considering these functions contained numerous overlapping genes, the functions were merged to produce a broader view of the genes involved. Furthermore, to examine the networks associated with acupuncture manipulation, IPA network analysis of the dysregulated genes in the hypothalami of the TRDM, TRFM and EA groups was performed. The analysis identified two networks: Cellular Assembly and Organization, Nervous System Development and Function,
Neurological Disease (CNN) with 24 focus genes (Fig. 2C, Table SIV) and Cellular Assembly and Organization, Cellular Function and Maintenance, Molecular Transport (CCM) with 19 focus genes (Fig. 2D, Table SIV) were identified. The CNN network was modulated by c-Jun, insulin, p38 mitogenactivated protein kinase, PI3K complex and cytokine, while the CCM network was modulated by Akt, AMPK, MTORC, PP2A, mTOR and STAT. Networks including 'Psychological Disorders', 'Nervous System Development and Function' and 'Neurological Disease' were assessed (Table SV). Multiple disease-related functions and networks affected by acupuncture treatments were identified.

Validation of the dysregulated proteins via PRM. Several proteins, including pre-mRNA-processing factor 6 (PRP6), heme binding protein 2 isoform CRA b (D3ZHC4), glucose-fructose oxidoreductase domain-containing 1 (D3ZR63), cell adhesion molecule L1-like (M0RC17), protein O-linked-mannose $\beta$-1,4-N-acetylglucosaminyltransferase 2 (PMGT2), RAB10 member RAS oncogene family (Q5RKJ9), dual specificity tyrosine-phosphorylation-regulated kinase $1 \mathrm{~A}$ (DYR1A), neudesin (NENF), sorting nexin-1 (SNX1), phosphoserine phosphatase (SERB) and NADH dehydrogenase 1 $\alpha$-subcomplex assembly factor 4 (NDUF4) selected from the 


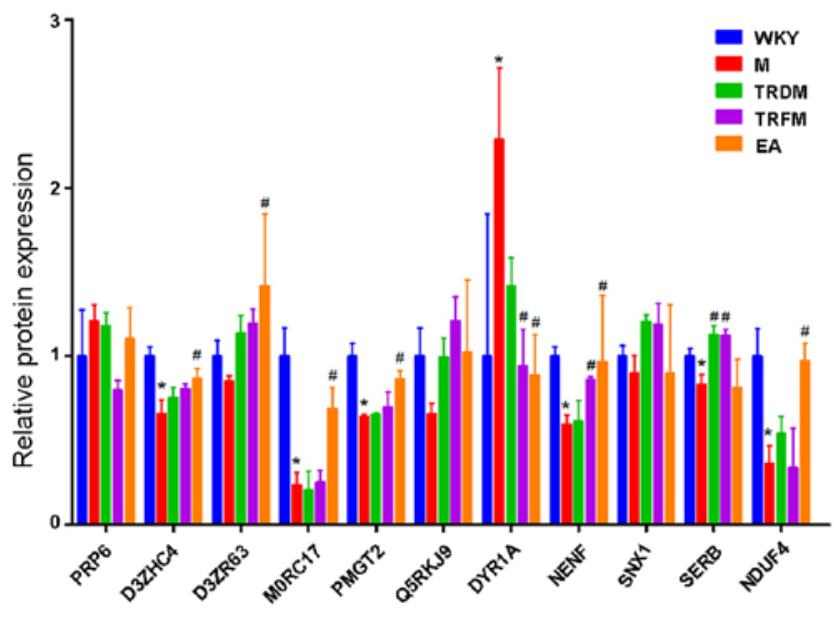

Figure 3. Protein expression profile of the protein candidates was validated by parallel reaction monitoring. ${ }^{*} \mathrm{P}<0.05$ vs. the WKY group. ${ }^{\text {"P }}<0.05$ vs. the $\mathrm{M}$ group. WKY, Wistar-Kyoto; $\mathrm{M}$, model; TRDM, twirling reducing manipulation; TRFM, twirling reinforcing manipulation; EA, electroacupuncture; PRP6, pre-mRNA-splicing factor 6; D3ZHC4, Heme binding protein 2 (Predicted), isoform CRA_b; D3ZR63, glucose-fructose oxidoreductase domain-containing 1; M0RC17, cell adhesion molecule L1-like, PMGT2, protein O-linked-mannose $\beta$-1,4-N-acetylglucosaminy 1transferase 2; Q5RKJ9, RAB10 member RAS oncogene family; DYR1A dual specificity tyrosine-phosphorylation-regulated kinase 1A; NENF, neudesin neurotrophic factor; SNX1, sorting nexin-1; SERB, phosphoserine phosphatase; NDUF4, NADH dehydrogenase [ubiquinone] $1 \alpha$ subcomplex assembly factor 4 .

GO enrichment and IPA analyses were subjected to validation by PRM (Fig. 3). D3ZHC4, MORC17, PMGT2, Q6IUR5, Q9NQR8 and Q5M819 in the hypothalami of the M group were significantly downregulated compared with the WKY group. EA manipulation rescued the expression of D3ZHC4, MORC17, PMGT2, Q6IUR5 and Q9NQR8 to varying extents. The TRFM group rescued Q6IUR5 and Q5M819. Furthermore, Q63470 was significantly upregulated in the M group compared with the WKY group and significantly downregulated in the TRFM and EA groups compared with Group M. However, NENF, a neurotrophic factor in neurons (39), was significantly upregulated in both TRFM and EA group compared with $\mathbf{M}$ group, indicating that acupuncture manipulation had an effect on the central nervous system.

\section{Discussion}

Numerous experimental and case studies have indicated that acupuncture manipulation lowered the blood pressure measurements of SHRs and patients with hypertension $(23,40,41)$ and LR3 was the most frequently used location in clinical trials $(42,43)$. In previous studies, TRDM, TRFM and EA at the LR3 location lowered the blood pressure measurements of SHRs and affected vascular smooth muscle through a central mechanism involving activated key brain regions $(35,44)$. Several studies have demonstrated that LR3 affects biological processes via the hypothalamus (5-47). Acupuncture manipulation on LR3 has been reported to inhibit the hyperactive hypothalamic-pituitary-adrenal axis in depression treatments (45). Luo et al (46) demonstrated that needling at LR3 may reduce blood pressure in renal canaliculi by altering the number of cells in the hypothalamus and the expression of glucose transporter 1. The twirling reinforcing-reducing manipulation (TRRM) treatment in LR3 significantly lowered the blood pressure and protected hypothalamic neurons by positively regulating the expression of the hypothalamic renin-angiotensin system (RAS) components of SHRs, which may be one of the central mechanisms by which TRRM induces antihypertensive effects (47).

In the present study, the antihypertensive effects of three acupuncture manipulations (TRDM, TRFM and EA) on LR3 were compared. To further reveal the central mechanism underlying the antihypertensive effect of acupuncture treatments, proteomics analysis was performed to examine the protein expression in the hypothalamus, followed by a PRM experiment for validation. The results demonstrated that different acupuncture treatments lowered blood pressure to varying degrees and multiple dysregulated proteins and potential networks associated with acupuncture stimuli were identified.

A series of blood pressure tests demonstrated that all three acupuncture manipulations used in the current study exhibited antihypertensive effects to varying degrees. The blood pressure measurements of the SHRs constantly rose compared with WKY rats. As indicated in Table I, TRFM and EA stimuli of SHRs reduced the blood pressure in comparison to that of the $\mathrm{M}$ group from day 8 . A continuous decline trend in the blood pressure of TRDM group was identified and the blood pressure measurements in the TRDM group were the lowest of all groups (M, EA and TRFM) at the end of the experiment, indicating that stimulating LR3 by TRDM exhibited the highest efficacy. The decreased blood pressure by acupuncture manipulations varied with the acupuncture methods and, for the LR3 loci, TRDM was the most effective in reducing blood pressure. Furthermore, the LI11 (Quchi), GB20 (Fengchi), ST36 (Zusanli), ST40 (Fenglong), LI4 (Hegu) and ST9 (Renying) points have been used in the clinical treatment for hypertension $(48,49)$. Further studies are required to investigate the antihypertensive effects of different acupuncture manipulations on different acupuncture points.

The central nervous system, particularly the hypothalamus, influences the regulation of the cardiovascular system and the hypertension development (27). Gene expression, including expression of mRNAs and microRNAs, in response to acupuncture therapy has been previously studied $(28,50)$. Furthermore, it has been hypothesized that protein expression levels are partially associated with transcript levels (51). A previous study has indicated that acupuncture manipulation at LR3 influences cerebral glucose metabolism in multiple brain regions, including the cerebellum, hypothalamus, midbrain and hippocampus (34). To further investigate the effect of acupuncture manipulation at LR3 on hypothalamus, the protein expression profiles in the hypothalamus were examined and GO and IPA were used for data mining. The results demonstrated that numerous secreted proteins participated in the antihypertensive mechanism in the hypothalamus and that certain secreted proteins (Table SV). The results also revealed that NENF, was downregulated in the hypothalamus of the SHRs compared with the WKY rats and that SHRs receiving acupuncture treatments exhibited reversal of the downregulation of NENF. NENF, a secreted protein, serves a role in the development of obesity, diabetes and melanoma (52-55), and may therefore represent a potential drug target. While 
it has been demonstrated that the neurotrophic mechanism of NENF is dependent on heme binding activity, the exact pathway remains to be elucidated (54). A previous study reported that NENF is associated with protein deglycase DJ-1 and PTEN-induced kinase 1 (PINK1) and that this association may promote neurotrophic activity and neuronal survival (55). Additionally, NENF has been reported to serve as an anorexigenic factor in the hypothalamus (56). Due to this, NENF may represent a potential target for drugs to treat hypertension. Furthermore, 10 more neuron developmentand neuritogenesis-related proteins were identified; A2MG, ACY2, DVL1, DYR1A, FNTA, KCJ10, KDIS, COLI, RAB10 and SNX1 (Table SIII). Further research is required to address the association between NENF and these proteins, with regards to neurotrophic activity. In future studies the expression of DJ-1 and PINK1 will be analyzed in the hypothalami of acupuncture-treated SHRs to evaluate the antihypertensive effect of hypothalamic DJ-1 and PINK1. To fully elucidate the mechanism of the antihypertensive effect of acupuncture manipulation, further research on protein expression analysis in the hypothalamus and other brain regions is required.

In conclusion, the results of the current study demonstrated that TRDM, TRFM and EA manipulations at the LR3 point reduced blood pressure measurements and that TRDM was the most effective of the techniques used. The hypothalamic protein expression profiles of the SHRs were altered following acupuncture therapy. Furthermore, multiple networks and proteins, particularly NENF, were identified and they may represent potential antihypertensive drug targets.

\section{Acknowledgements}

Not applicable.

\section{Funding}

The current study was supported by Chinese Nature Science Foundation Grants (grant no. 81774413).

\section{Availability of data and materials}

All the thermo raw files of proteomics analysis can be obtained from iProx database (https://www.iprox.org/page/HMV006. html, accession number IPX0001978001). Additional data is available from the corresponding author on reasonable request.

\section{Authors' contributions}

JRL wrote the paper and was responsible for the design and running of the main experiments. QGL was involved in the design of the study. JJW and XDZ measured the blood pressure of rats. XMH, TXZ and JS performed acupuncture manipulations. ZJ, KP and KML analyzed the data. All authors read and approved the final maunscript.

\section{Ethics approval and consent to participate}

All experimental procedures were approved by the Animal Care and Use Committee of Beijing University of Chinese Medicine, Beijing, China (permit no. BUCM-3-2016090301-3003).

\section{Patient consent for publication}

Not applicable.

\section{Competing interests}

The authors declare that they have no competing interests.

\section{References}

1. Bloch MJ: Worldwide prevalence of hypertension exceeds 1.3 billion. J Am Soc Hypertens 10: 753-754, 2016.

2. Messerli FH, Williams B and Ritz E: Essential hypertension. Lancet 370: 591-603, 2007.

3. Garfinkle MA: Salt and essential hypertension: Pathophysiology and implications for treatment. J Am Soc Hypertens 11: 385-391, 2017.

4. Sousa AC, Mendonça MI, Pereira A, Gouveia S, Freitas AI, Guerra G, Rodrigues M, Henriques E, Freitas S, Borges S, et al: Synergistic association of genetic variants with environmental risk factors in susceptibility to essential hypertension. Genet Test Mol Biomarkers 21: 625-631, 2017.

5. Wrzosek M, Sokal M, Sawicka A, Wlodarczyk M, Glowala M, Wrzosek M, Kosior M, Talalaj M, Biecek P and Nowicka G: Impact of obesity and nitric oxide synthase gene G894T polymorphism on essential hypertension. J Physiol Pharmacol 66: 681-689, 2015.

6. Fonkoue IT, Wang M and Carter JR: Sympathetic neural reactivity to mental stress in offspring of hypertensive parents: 20 Years revisited. Am J Physiol Heart Circ Physiol 311: H426-H432, 2016.

7. Mensah GA, Croft JB and Giles WH: The heart, kidney, and brain as target organs in hypertension. Curr Probl Cardiol 28: 156-193, 2003.

8. Azizi M, Rossignol P and Hulot JS: Emerging drug classes and their potential use in hypertension. Hypertension 74: 1075-1083, 2019.

9. Gniwa Omezzine R, Akkara A, Abdelkafi Koubaa A, Belguith Sriha A, Rdissi A and Amamou K: Predictors of poor adherence to hypertension treatment. Tunis Med 97: 564-571, 2019.

10. Nagayama T, Nishida M, Hizue M, Ogino Y and Fujiyoshi M: Adverse drug reactions for medicines newly approved in japan from 1999 to 2013: Hypertension and hypotension. Basic Clin Pharmacol Toxicol 118: 306-312, 2016.

11. Li X, Zhang L, Jian L, Li L and Niu S: Curative effect of amlodipine combined with enalapril in curing hypertension of the aged. Pak J Pharm Sci 28 (2 Suppl): S693-S696, 2015.

12. Flachskampf FA, Gallasch J, Gefeller O, Gan J, Mao J, Pfahlberg AB, Wortmann A, Klinghammer L, Pflederer W and Daniel WG: Randomized trial of acupuncture to lower blood pressure. Circulation 115: 3121-3129, 2007.

13. Turnbull $\mathrm{F}$ and Patel A: Acupuncture for blood pressure lowering: Needling the truth. Circulation 115: 3048-3049, 2007.

14. Tjen-A-Looi SC and Longhurst CA: John C. Longhurst, MD, PhD (1947-2018): A pioneer in acupuncture hypertension research. Am J Physiol Heart Circ Physiol 314: H1153-H1154, 2018.

15. Zhuang Y, Xing JJ, Li J, Zeng BY and Liang FR: History of acupuncture research. Int Rev Neurobiol 111: 1-23, 2013.

16. He JL: Preliminary discussion on the theory of body physiognomy in the Huangdineijing (Huangdi's Inner Classic). Zhonghua Yi Shi Za Zhi 39: 189-192, 2009 (In Chinese).

17. Zhu DC, Leng C, Xiong J and Ye WG: Thermosensitive moxibustion induces a better therapeutic effect in the treatment of facial paralysis patients. Zhen Ci Yan Jiu 43: 666-669, 2018 (In Chinese).

18. Xu L, Jing L, He K, Wang JL and Wang Y: Treatment of knee osteoarthritis with acupuncture and moxibustion: A randomized controlled trial. Zhongguo Zhen Jiu 33: 871-876, 2013 (In Chinese).

19. Liu W, Zhu LQ, Chen SS, Lu SC, Tang J and Liu QG: Effect of twirling-reinforcing-reducing needling manipulations on contents of serum acetylcholine and arterial NOS and cGMP in stress-induced hypertension rats. Zhen Ci Yan Jiu 40: 136-140, 2015 (In Chinese).

20. Chen H, Dai J, Zhang X, Wang K, Huang S, Cao Q, Wang H, Liang Y, Shi C, Li M, et al: Hypothalamus-related resting brain network underlying short-term acupuncture treatment in primary hypertension. Evid Based Complement Alternat Med 2013: 808971, 2013. 
21. Cheng L, Li P, Tjen-A-Looi SC and Longhurst JC: What do we understand from clinical and mechanistic studies on acupuncture treatment for hypertension? Chin Med 10: 36, 2015.

22. Li J, Wang Y, He K, Peng C, Wu P, Li C and Lai X: Effect of acupuncture at LR3 on cerebral glucose metabolism in a rat model of hypertension: A ${ }^{18} \mathrm{~F}-\mathrm{FDG}-\mathrm{PET}$ study. Evid Based Complement Alternat Med 2018: 5712857, 2018.

23. Lai X, Wang J, Nabar NR, Pan S, Tang C, Huang Y, Hao M, Yang Z, Ma C, Zhang J, et al: Proteomic response to acupuncture treatment in spontaneously hypertensive rats. PLoS One 7: e44216, 2012.

24. Li J, Sun M, Ye J, Li Y, Jin R, Zheng H and Liang F: The mechanism of acupuncture in treating essential hypertension: A narrative review. Int J Hypertens 2019: 8676490, 2019.

25. Santisteban MM, Kim S, Pepine CJ and Raizada MK: Brain-gut-bone marrow axis: Implications for hypertension and related therapeutics. Circ Res 118: 1327-1336, 2016.

26. Feng XF and Ma TM: Compared with twirling and rotating acupuncture manipulations in similarities and differences. J Liaoning Univ Traditi Chin Med 12: No. 10, 2010 (In Chinese)

27. Khor S and Cai D: Hypothalamic and inflammatory basis of hypertension. Clin Sci (Lond) 131: 211-223, 2017.

28. Ma SM, Yang JW, Tu JF, Yang NN, Du YZ, Wang XR, Wang L, Huang J and Liu CZ: Gene-level regulation of acupuncture therapy in spontaneously hypertensive rats: A whole transcriptome analysis. Evid Based Complement Alternat Med 2019: 9541079, 2019.

29. Yang JW, Ye Y, Wang XR, Li F, Xiao LY, Shi GX and Liu CZ: Acupuncture attenuates renal sympathetic activity and blood pressure via beta-adrenergic receptors in spontaneously hypertensive rats. Neural Plast 2017: 8696402, 2017.

30. Okamoto $\mathrm{K}$ and Aoki K: Development of a strain of spontaneously hypertensive rats. Jpn Circ J 27: 282-23, 1963.

31. Jones-Bolin S: Guidelines for the care and use of laboratory animals in biomedical research. Curr Protoc Pharmacol 59: A.4B.1-A.4B.9, 2012.

32. Conn PM (ed): Animal models for the study of human disease. 2nd edition. Academic Press, pp19, 1177, 2017.

33. Okamoto K, Tabei R, Fukushima M, Nosaka S and Yamori Y: Further observations of the development of a strain of spontaneously hypertensive rats. Jpn Circ J 30: 703-716, 1966.

34. Guo Q, Liu Q, Sun D and Nie B: Twirling reinforcing-reducing manipulation-central mechanism underlying antihypertensive effect on spontaneous hypertension in rats. J Tradit Chin Med 38: 391-398, 2018

35. Guo Y, Xie X, Guo C, Wang Z and Liu Q: Effect of electro-acupuncture on gene expression in heart of rats with stress-induced pre-hypertension based on gene chip technology. J Tradit Chin Med 35: 285-294, 2015.

36. Yi-Ming W, Shu H, Miao CY, Shen FM, Jiang YY and Su DF Asynchronism of the recovery of baroreflex sensitivity, blood pressure, and consciousness from anesthesia in rats. J Cardiovasc Pharmacol 43: 1-7, 2004.

37. Redfors B, Shao Y and Omerovic E: Influence of anesthetic agent, depth of anesthesia and body temperature on cardiovascular functional parameters in the rat. Lab Anim 48: 6-14, 2014.

38. Xiang B, Zhong P, Fang L, Wu X, Song Y, and Yuan H: miR-183 inhibits microglia activation and expression of inflammatory factors in rats with cerebral ischemia reperfusion via $N F-\kappa B$ signaling pathway. Exp Ther Med 18: 2540-2546, 2019.

39. Moutaoufik MT, Malty R, Amin S, Zhang Q, Phanse S Gagarinova A,Zilocchi M, Hoell L, Minic Z, Gagarinova M, et al: Rewiring of the human mitochondrial interactome during neuronal reprogramming reveals regulators of the respirasome and neurogenesis. iScience 19: 1114-1132, 2019.
40. Chiu YJ, Chi A and Reid IA: Cardiovascular and endocrine effects of acupuncture in hypertensive patients. Clin Exp Hypertens 19: 1047-1063, 1997.

41. Wang J, Xiong X and Liu W: Acupuncture for essential hypertension. Int J Cardiol 169: 317-326, 2013.

42. Zhao XF, Hu HT, Li JS, Shang HC, Zheng HZ, Niu JF, Shi XM and Wang S: Is acupuncture effective for hypertension? A systematic review and meta-analysis. PLoS One 10: e0127019, 2015.

43. Wang Y, Zheng Y, Qu S, Zhang J, Zhong Z, Zhang J, Huang H, Li M, Xu Y, Chen J, et al: Cerebral targeting of acupuncture at combined acupoints in treating essential hypertension: An Rs-fMRI study and curative effect evidence. Evid Based Complement Alternat Med 2016: 5392954, 2016.

44. Qiulei G: Twirling reinforcing-reducing manipulation on upoints in treating essential hypertension: An Rs-fMRn spontaneous hypertension in rats. J Tradit Chin Med 38: 391-398, 2018.

45. Le JJ, Yi T, Qi L, Li J, Shao L and Dong JC: Electroacupuncture regulate hypothalamic-pituitary-adrenal axis and enhance hippocampal serotonin system in a rat model of depression. Neurosci Lett 615: 66-71, 2016.

46. Luo X, Huang J, Yu J and nTang C: Effect of Taichong (LR 3) acupuncture in spontaneously hypertensive rats. J Tradit Chin Med 39: 74-80, 2019.

47. Amlie-Lefond C, Sébire G and Fullerton HJ: Recent developments in childhood arterial ischaemic stroke. Lancet Neurol 7: 425-435, 2008.

48. Zhang L, Shen P and Wang S: Acupuncture treatment for hypertension: A case study. Acupunct Med 32: 73-76, 2014

49. Lee H, Kim SY, Park J, Kim YJ, Lee H and Park HJ: Acupuncture for lowering blood pressure: Systematic review and metaanalysis. Am J Hypertens 22: 122-128, 2009.

50. Wang JY, Li H, Ma CM, Wang JL, Lai XS and Zhou SF: MicroRNA profiling response to acupuncture therapy in spontaneously hypertensive rats. Evid Based Complement Alternat Med 2015: 204367, 2015.

51. Liu Y, Beyer A and Aebersold R: On the dependency of cellular protein levels on mRNA abundance. Cell 165: 535-550, 2016.

52. Kratochvilova H, Lacinova Z, Klouckova J, Kavalkova P, Cinkajzlova A, Trachta P, Krizova J, Benes M, Dolezalova K, Fried M, et al: Neudesin in obesity and type 2 diabetes mellitus: The effect of acute fasting and weight reducing interventions. Diabetes Metab Syndr Obes 12: 423-430, 2019.

53. Polkowska A, Pasierowska IE, Pasławska M, Pawluczuk E and Bossowski A: Assessment of serum concentrations of adropin, afamin, and neudesin in children with type 1 diabetes. Biomed Res Int 2019: 6128410, 2019.

54. Ortega-Bernal D, La Rosa CHG, Arechaga-Ocampo E, Alvarez-Avitia MA, Moreno NS and Rangel-Escareño C: A meta-analysis of transcriptome datasets characterizes malignant transformation from melanocytes and nevi to melanoma. Oncol Lett 16: 1899-1911, 2018.

55. Kimura I, Nakayama Y, Zhao Y, Konishi M and Itoh N: Neurotrophic effects of neudesin in the central nervous system. Front Neurosci 7: 111, 2013.

56. Byerly MS, Swanson RD, Semsarzadeh NN, McCulloh PS, Kwon K, Aja S, Moran TH, Wong GW and Blackshaw S: Identification of hypothalamic neuron-derived neurotrophic factor as a novel factor modulating appetite. Am J Physiol Regul Integr Comp Physiol 304: R1085-R1095, 2013.

This work is licensed under a Creative Commons Attribution-NonCommercial-NoDerivatives 4.0 International (CC BY-NC-ND 4.0) License. 